\title{
Do fluorescence and transient absorption probe the same intramolecular charge transfer state of 4-(dimethylamino)benzonitrile?
}

\author{
Thomas Gustavsson, ${ }^{1}$ Pedro B. Coto, ${ }^{2}$ Luis Serrano-Andrés, ${ }^{2}$ Takashige Fujiwara, ${ }^{3}$ and \\ Edward C. Lim ${ }^{3, a)}$ \\ ${ }^{1}$ Laboratoire Francis Perrin, CEA/DSM/DRECAM/SPAM-CNRS URA 2453, CEA/Saclay, \\ 91191 Gif-sur-Yvette Cedex, France \\ ${ }_{2}^{2}$ Molecular Science Institute, University of Valencia, P.O. Box 22085, ES-46071 Valencia, Spain \\ ${ }^{3}$ Department of Chemistry and The Center for Laser and Optical Spectroscopy, The University of Akron, \\ Akron, Ohio 44325-3601, USA
}

(Received 4 May 2009; accepted 18 June 2009; published online 15 July 2009)

\begin{abstract}
We present here the results of time-resolved absorption and emission experiments for 4-(dimethylamino)benzonitrile in solution, which suggest that the fluorescent intramolecular charge transfer (ICT) state may differ from the twisted ICT (TICT) state observed in transient absorption. (c) 2009 American Institute of Physics. [DOI: 10.1063/1.3173609]
\end{abstract}

Intramolecular charge transfer (ICT) in photoexcited 4-(dimethylamino)benzonitrile (DMABN) and related electron donor-acceptor molecules in polar solvents, leading to the appearance of dual fluorescence, ${ }^{1}$ has been a topic of very extensive experimental and computational research for several decades. ${ }^{2}$ Much of these studies have focused on the two most fundamental issues associated with the photoinduced ICT, namely, the geometrical structure of the ICT state and the electronic pathways that take the photoexcited DMABN (in the $\pi \pi^{*}$ state) to the ICT state.

The experimental probe of the ICT-state geometry has been made by studying fluorescence of covalently modified DMABN in which the electron-donor moiety (viz., dimethylamino group) is fixed to lie either parallel or perpendicular to the plane of the electron-acceptor moiety (viz., benzonitrile). These studies have established that the most likely geometry of the ICT state of DMABN is the so-called twisted ICT (TICT) state st $^{3,4}$ in which the positively charged dimethylamino group is twisted with respect to the plane of the negatively charged benzonitrile moiety. The twisted geometry minimizes the Coulombic interaction between the unpaired electrons with consequent stabilization of the ICT state. Consistent with the TICT model, the ICT-state absorptions at about 420 and $320 \mathrm{~nm}$ very closely resemble the absorption spectrum of the benzonitrile radical anion in both peak positions and relative intensities. ${ }^{5}$ Moreover, picosecond time-resolved resonance Raman spectra of the ICT state, obtained by using probe (Raman inducing) wavelength of $330 \mathrm{~nm}$, exhibit several characteristic modes that are very similar to the modes observed for the benzonitrile radical anion. ${ }^{6}$ An alternative ICT-state structure, known as PICT (P for planar), ${ }^{7}$ is not supported by time-resolved laser spectroscopies (vide infra) or by a great majority of quantum chemical calculations. ${ }^{8,9}$

The second issue, concerning the mechanism of the ICT reaction, has mostly been addressed by investigating the temporal characteristics of the fluorescence from the $S_{1}\left(\pi \pi^{*}\right)$, or

${ }^{a)}$ Electronic mail: elim@uakron.edu. the so-called locally excited (LE) state, with those of the longer-wavelength fluorescence from the ICT state. The results of the time-resolved picosecond fluorescence measurements for DMABN have indicated that the fast-decaying component of the LE emission has picosecond decay time, which is identical to the rise time of the ICT emission. ${ }^{10}$ Furthermore, the ICT emission was reported to have the same nanosecond decay time as the slow-decaying component of the LE emission. These observations led to the twostate conversion mechanism with the picosecond time constant corresponding the LE-ICT equilibration time and the nanosecond decay to the equilibrium population decay. ${ }^{10}$

While the two-state ICT reaction model is certainly very plausible, the primary experimental support for the mechanism is based on picosecond time-correlated single-photon counting, which has instrument response functions ( $\geq 20 \mathrm{ps}$ ) substantially longer than the decay time $(\sim 4 \mathrm{ps})$ of the fast-decaying component of the LE fluorescence. Temporal measurements with much superior time resolution are therefore needed to rigorously test the validity of the reaction mechanism. To complicate the matter further, recent femtosecond transient absorption measurements strongly suggest that the fully charge-separated (zwitterionic) ICT state is formed from the $\pi \sigma^{*}$ state. $^{11}$ Thus, the well-known benzonitrile-radical-anion-like absorption of the TICT state at about 320 and $420 \mathrm{~nm}$ have rise time $(\sim 4.1 \mathrm{ps})$ that is identical to the decay time of the $700 \mathrm{~nm} \pi \sigma^{*}$ state transient, for DMABN in acetonitrile at room temperature. The observed kinetics is consistent with a sequential ICT process in which the initially excited $S_{2}\left(\pi \pi^{*}\right)$ state of $L_{a}$ character produces the TICT state via the intermediate electronic state of $\pi \sigma_{\mathrm{CN}}^{*}$ electron configuration. ${ }^{11-13}$ There are also observations that the decay time of the long-lived component of the LE fluorescence is not identical to the decay time of the ICT fluorescence, with the former being shorter for dialkylaminobenzonitriles. ${ }^{14-17}$ These results strongly indicate that the actual mechanism of the ICT reaction in DMABN may be far more complex than previously assumed. 

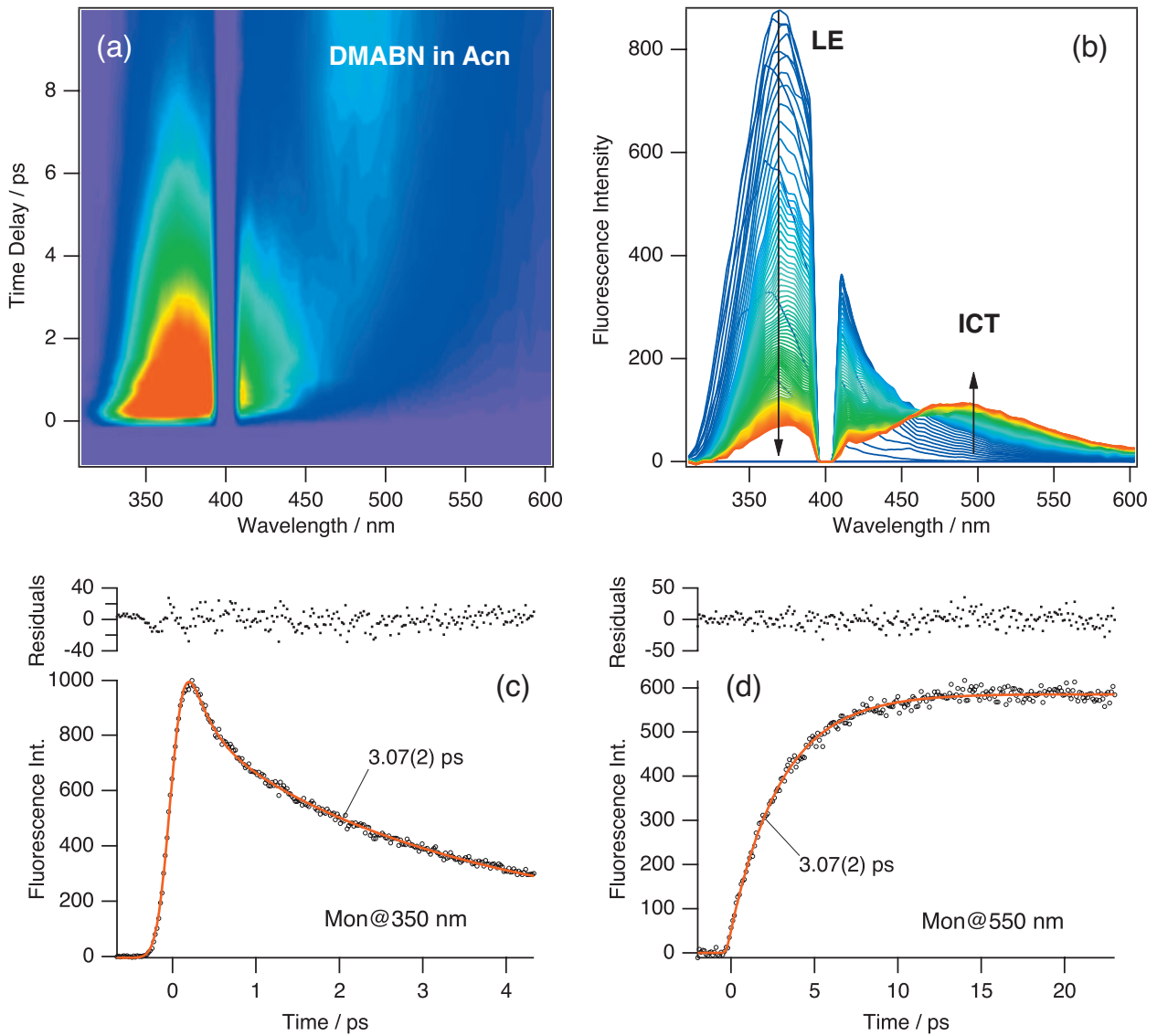

(c)
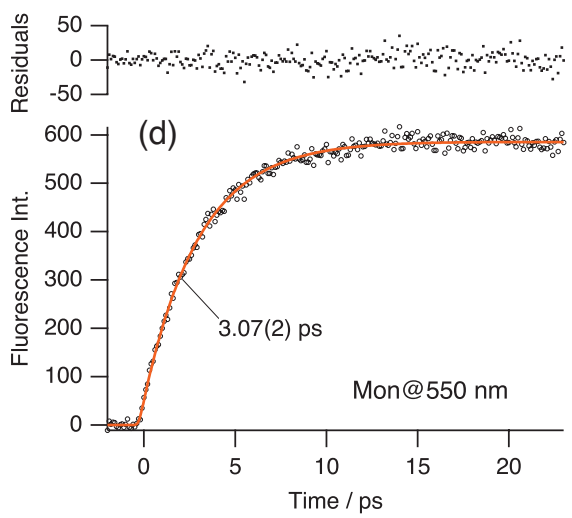

FIG. 1. The femtosecond timeresolved fluorescence upconversion spectra: (a) two-dimensional image plot and (b) its corresponding timeevoluted spectra of DMABN in acetonitrile (Acn) at room temperature. The spectral region around $400 \mathrm{~nm}$ was masked thoroughly to protect a detector in the excitation wavelength of $267 \mathrm{~nm}$. The fluorescence decay curves of (c) the LE emission at $350 \mathrm{~nm}$ and (d) the ICT emission at $550 \mathrm{~nm}$ for DMABN in Acn. Common decay time constants were evaluated including other upconversion sets (not shown here) by a nonlinear leastsquares global fit with a deconvolution procedure. The numbers in the parentheses indicate a standard deviation $(1 \sigma)$.
In an effort to develop a more detailed picture of the ICT reaction in dialkylaminobenzonitriles, we have been carrying out a concerted experimental and ab initio computational study of the reaction pathways that lead photoexcited DMABN to the TICT state. In this communication, we present the results of time-resolved absorption ${ }^{18}$ and fluorescence upconversion ${ }^{19}$ experiments for DMABN in acetonitrile and ethanol, which suggest that the fluorescent ICT state observed in emission differs from the zwitterionic TICT state observed in transient absorption.

Figure 1 presents the temporal characteristics of fluorescence of DMABN in acetonitrile at room temperature, as measured by fluorescence upconversion. To greatly reduce the effect of photoproduct formation (a loss of a methyl group to produce 4-methylaminobenzonitrile ${ }^{20}$ ), the steadystate absorption and fluorescence spectra were measured before and after the upconversion measurement, to ensure that only single species (DMABN) was monitored in the upconversion signal. It is evident from the figure that the rise time $(\sim 3 \mathrm{ps})$ of the ICT emission at about $550 \mathrm{~nm}$ is very similar to the decay time $(\sim 3 \mathrm{ps})$ of the LE fluorescence, thus confirming the precursor-successor relationship between the LE and the ICT states. The time-resolved fluorescence upconversion signal at $350 \mathrm{~nm}$, in the pump-gate delay times of 0-8 ps, can be fitted with a double exponential function, with a subpicosecond decay followed by the 3 ps decay. The subpicosecond LE upconversion transient is believed to be related to solvation dynamics leading to a spectral shift, which is very rapid ( $\sim 0.26$ ps correlation time) (Ref. 21$)$ in acetonitrile. Common decay time constants were evaluated includ- ing other upconversion data taken at a variety of observed wavelengths by nonlinear least-squares fitting with deconvolution procedure. Table I summarizes global fit analyses in which some of decay constants were considered as global parameters while others were all varied. The global fitting was successfully converged and found the common decay constants.

Figure 2 compares the picosecond rise time of the TICTstate absorption at $420 \mathrm{~nm}$ with the decay time of the $\pi \sigma^{*}$-state absorption at $680 \mathrm{~nm}$. At both 680 and $420 \mathrm{~nm}$, stimulated emission and ground-state bleach are too small to affect the kinetics of the transients. The ground-state bleach, for instance, starts below $320 \mathrm{~nm}$ where the WLC probe also reaches to its blue edge of the profile. Thus, as a result of mutual changes the observed transient absorption spectra at the blue appear somewhat flat. The identity of the two time constants $(\sim 4.3 \mathrm{ps})$ demonstrates the formation of the TICT state from the $\pi \sigma^{*}$ state. The rise time of the TICT-state absorption is longer than that of the ICT emission ( $3 \mathrm{ps})$. The differing rise times suggest that the emissive ICT state, responsible for the redshifted fluorescence, may be different from the TICT state observed in the transient absorption.

In Fig. 3, the nanosecond decay time $(\sim 2.9 \mathrm{~ns})$ of the $470 \mathrm{~nm}$ ICT fluorescence is compared to the nanosecond decay time $(\sim 4.8 \mathrm{~ns})$ of the $330 \mathrm{~nm}$ TICT-state absorption ${ }^{22}$ for DMABN in acetonitrile. It is evident from the results that the decay time of the TICT-state absorption at $330 \mathrm{~nm}$ is substantially longer than the decay time of the ICT emission. As the lifetime of the ICT emission is determined mostly by its nonradiative decay process, $\left[k_{\mathrm{r}}=0.0079 \times 10^{9} \mathrm{~s}^{-1}\right.$ 
TABLE I. Global fit analyses in the fluorescence upconversion measurements of DMABN/Acn at $\lambda_{\mathrm{exc}}$ $=267 \mathrm{~nm}$. All decay constants are in ps, if not otherwise stated.

\begin{tabular}{|c|c|c|c|c|c|c|c|}
\hline $\begin{array}{l}\text { Observed } \\
\text { wavelength }\end{array}$ & $325 \mathrm{~nm}$ & $350 \mathrm{~nm}$ & $450 \mathrm{~nm}$ & $500 \mathrm{~nm}$ & $550 \mathrm{~nm}$ & $575 \mathrm{~nm}$ & $600 \mathrm{~nm}$ \\
\hline$\tau_{1}^{\mathrm{a}}$ & 0.239 & 0.239 & 0.239 (rise) & & & & \\
\hline $\begin{array}{l}\tau_{2}^{\mathrm{a}} \\
\tau_{3}{ }^{\mathrm{a}}\end{array}$ & 3.067 & 3.067 & $\begin{array}{c}3.067 \\
169.8^{\mathrm{b}}\end{array}$ & 3.067 (rise) & 3.067 (rise) & 3.067 (rise) & 3.067 (rise) \\
\hline FWHM $^{\mathrm{c}}$ & $286 \mathrm{fs}$ & $333 \mathrm{fs}$ & $332 \mathrm{fs}$ & $301 \mathrm{fs}$ & $301 \mathrm{fs}$ & $301 \mathrm{fs}$ & $301 \mathrm{fs}$ \\
\hline No. of run ${ }^{d}$ & 2 & 3 & 3 & 1 & 4 & 1 & 1 \\
\hline
\end{tabular}

accuracies of the common decay constant of $\tau_{1}, \tau_{2}$, and $\tau_{3}$ are $\pm 0.003, \pm 0.024$, and $\pm 25.6 \mathrm{ps}$, respectively.

${ }^{\mathrm{b}}$ It may appear a nominal decay constant since the LE and ICT bands overlap in this spectral regime, which also accounts for having a large inaccuracy in $\tau_{3}$.

${ }^{\mathrm{c}}$ Full width at half maximum of a Gaussian function used as the system response in the fit.

${ }^{\mathrm{d}}$ Number of traces included in the global fit.

(Ref. 10) versus $k_{\mathrm{nr}}=0.2 \times 10^{9} \mathrm{~s}^{-1}$ ] the decay time of the ICT fluorescence should be essentially the same as that deduced by transient absorption, if only one ICT state is present in the molecule. Thus, the differing decay rates of the ICT fluorescence and the TICT state absorption again indicate that the two ICT states could be different. This conclusion also rationalizes the observation that 4-(diisopropylamino)benzonitrile, which exhibits the ICT fluorescence even in nonpolar solvents, does not display the $420 \mathrm{~nm}$ TICT transient in $n$-hexane. ${ }^{11}$ It is also significant that the decay time of the long-lived component of LE fluorescence is not identical to the decay time of the ICT fluorescence, Fig. 3(d).

Similar discrepancies between the time constants of the TICT-state absorption and the ICT fluorescence exist for DMABN in ethanol. Thus, the 9.9 ps rise time and the $3.3 \mathrm{~ns}$ decay time of the $420 \mathrm{~nm}$ TICT-state absorption are significantly greater than the $6.0 \mathrm{ps}$ rise time and 2.3 ns decay time of the ICT fluorescence at room temperature. It is possible that the slower picosecond rise time of the ICT states in ethanol, as compared to acetonitrile, is related to H-bonding in ethanol. ${ }^{23}$

To summarize, our data suggest the existence of two charge-transfer states of DMABN in polar solvents, namely, the dark TICT state formed form the $\pi \sigma^{*}$ state and the fluorescent ICT state formed from the LE state. Differing time scales of the two ICT states indicate the lack of communication (i.e., rapid population equilibration) between them. The understanding of the presence of the two noncommunicating ICT states requires an accurate characterization of the potential-energy profiles of the low-lying electronic states of
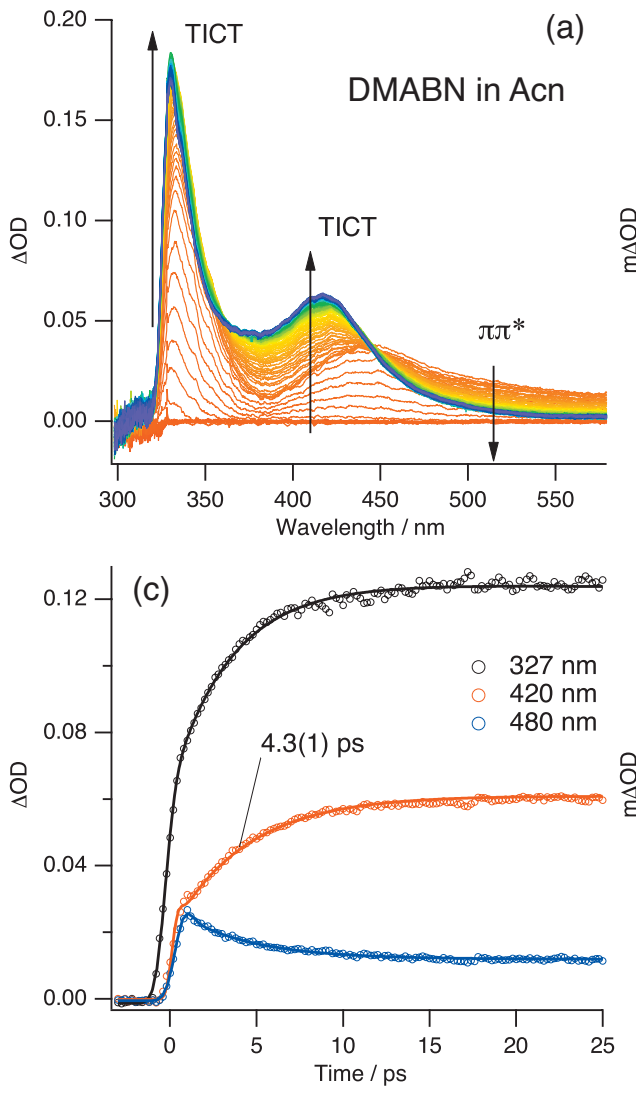
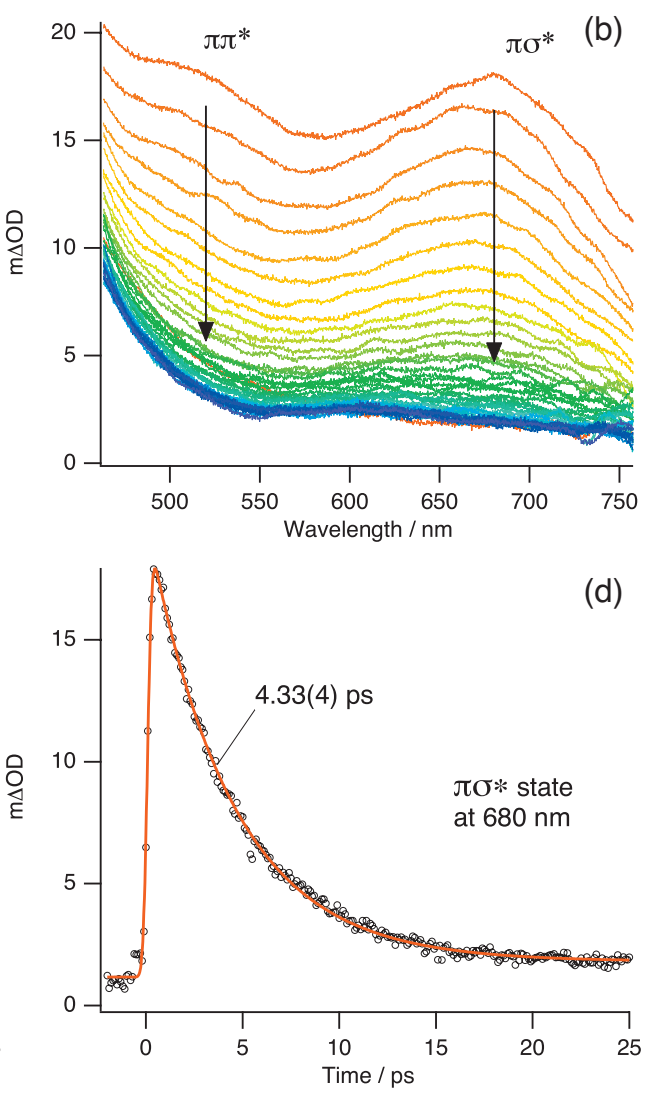

FIG. 2. The femtosecond timeresolved transient absorption spectra $(-2$ to 25 ps with an interval of $0.1 \mathrm{ps})$ at (a) shorter- and (b) longerwavelength region for DMABN in Acn at room temperature. The arrows indicate the time evolution of the spectra, and assignments for the transients are also shown. Kinetics at (c) the $420 \mathrm{~nm}$ transient (TICT) and (d) $680 \mathrm{~nm}$ transient (the $\pi \sigma^{*}$-state absorption), which were extracted with a band integration from the timeresolved spectra [(a) and (b)]. Leaders show the fitted decay times with a standard deviation in the parentheses. 

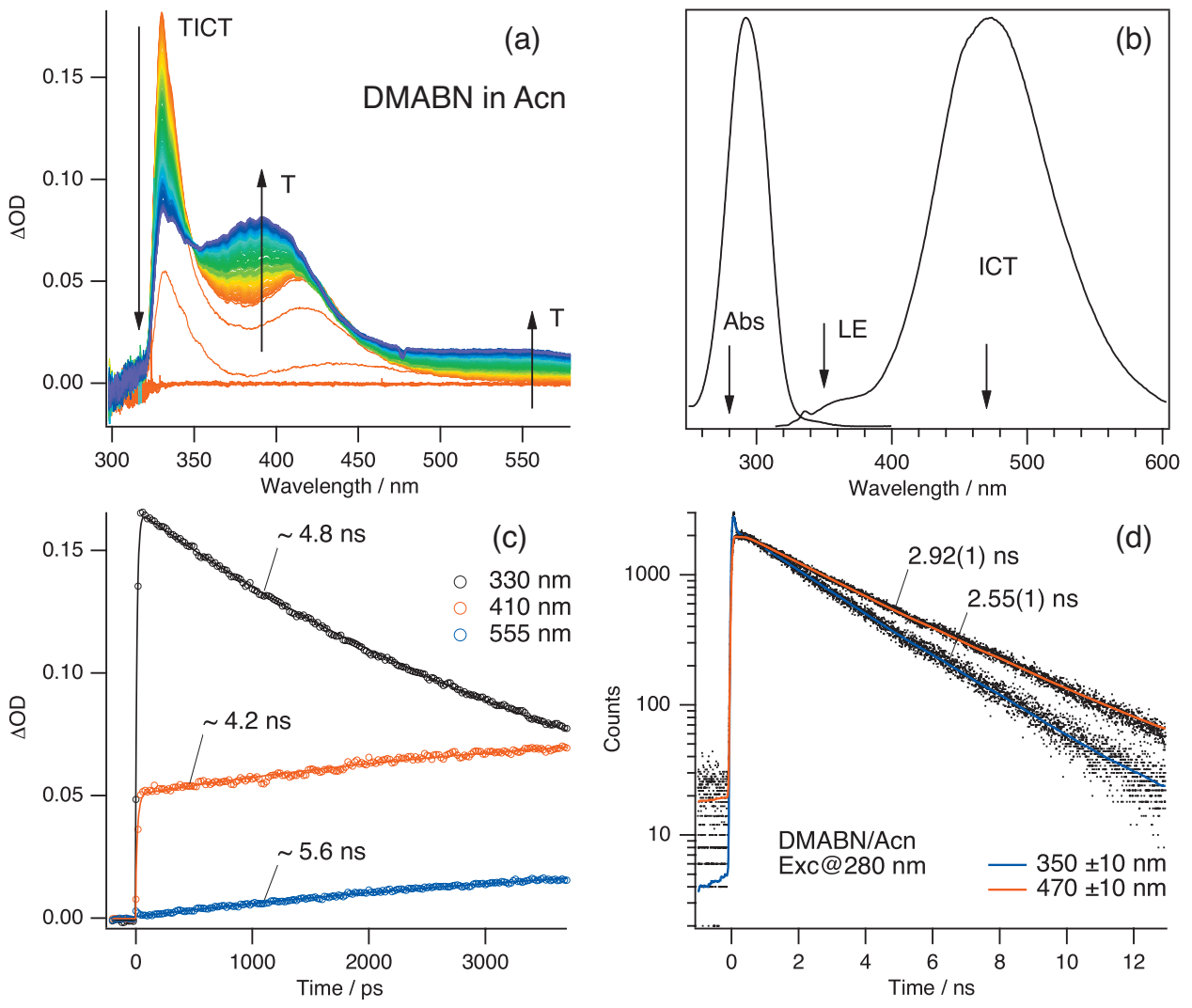

FIG. 3. Comparison of the long decay of the ICT fluorescence and the TICT transient absorption: (a) The time-resolved transient absorption spectra ( -0.2 to $3.7 \mathrm{~ns}$ with an interval of $20 \mathrm{ps}$ ) and (b) steady-state absorption and emission spectra for DMABN in Acn at room temperature. The arrows indicate the time evolution of the spectra, and annotation of T represents the triplet-triplet transient absorption. (c) Kinetics at the $330 \mathrm{~nm}$ TICT transient as well as those of 410 and $555 \mathrm{~nm}$ for DMABN in acetonitrile, generated with band integration of the time-resolved spectra (a). (d) Fluorescence decay curve of the ICT emission at $470 \mathrm{~nm}$, and that of the LE emission at $350 \mathrm{~nm}$ measured by a picosecond time-correlated single-photon counting in the excitation of $280 \mathrm{~nm}$. Both decay curves were fitted well with double exponential (fast and slow decay components). Leaders only present the slow decay time with a standard deviation in the parentheses to show the difference.

DMABN, using state-of-the-art $a b$ initio methods. A work along this line is presently in progress.

The work has received funding from the EC's Seventh Framework Programme (FP7/2007-2013) under Grant Agreement No. 228334, Contract No. 212025 (LASERLABEUROPE CONT.) and the French Programme "Triangle de la Physique." Financial support was also acknowledged from GV/2008/059 Generalitat Valenciana and Spanish MEC for Junan de la Cierva Programme, and from projects CTQ200761260 and CSD2007-0010 Consolider-Ingenio in Molecular Nanoscience of the Spanish MEC/FEDER, and Faculty Research Grant of The University of Akron.

${ }^{1}$ E. Lippert, W. Lüder, and H. Boos, in Advances in Molecular Spectroscopy, edited by A. Mangini (Pergamon, Oxford, 1962), p. 443.

${ }^{2}$ Z. R. Grabowski, K. Rotkiewicz, and W. Rettig, Chem. Rev. (Washington, D.C.) 103, 3899 (2003), and references therein.

${ }^{3}$ K. Rotkiewicz, K. H. Grellmann, and Z. R. Grabowski, Chem. Phys. Lett. 19, 315 (1973).

${ }^{4}$ Z. R. Grabowski, K. Rotkiewicz, A. Siemiarczuk, D. J. Cowley, and W. Baumann, Nouv. J. Chim. 3, 443 (1979).

${ }^{5}$ T. Okada, M. Uesugi, G. Kohler, K. Rechthaler, K. Rotkiewicz, W. Rettig, and G. Grabner, Chem. Phys. 241, 327 (1999).

${ }^{6}$ W. M. Kwok, C. Ma, P. Matousek, A. W. Parker, D. Phillips, W. T. Toner, M. Towrie, and S. Umapathy, J. Phys. Chem. A 105, 984 (2001).

${ }^{7}$ K. A. Zachariasse, S. I. Druzhinin, W. Bosch, and R. Machinek, J. Am. Chem. Soc. 126, 1705 (2004).
${ }^{8}$ C. Hättig, A. Hellweg, and A. Köhn, J. Am. Chem. Soc. 128, 15672 (2006).

${ }^{9}$ I. Gomez, Y. Mercier, and M. Reguero, J. Phys. Chem. A 110, 11455 (2006)

${ }^{10}$ S. I. Druzhinin, N. P. Ernsting, S. A. Kovalenko, L. P. Lustres, T. A. Senyushkina, and K. A. Zachariasse, J. Phys. Chem. A 110, 2955 (2006), and references therein.

${ }^{11}$ J.-K. Lee, T. Fujiwara, W. G. Kofron, M. Z. Zgierski, and E. C. Lim, J. Chem. Phys. 128, 164512 (2008).

${ }^{12}$ M. Z. Zgierski and E. C. Lim, J. Chem. Phys. 121, 2462 (2004).

${ }^{13}$ M. Z. Zgierski and E. C. Lim, J. Chem. Phys. 122, 111103 (2005).

${ }^{14}$ P. Changenet, P. Plaza, M. M. Martin, and Y. H. Meyer, J. Phys. Chem. A 101, 8186 (1997)

${ }^{15}$ E. Iwase, A. Tomioka, H. Saigusa, and M. Yagi, Phys. Chem. Chem. Phys. 6, 3852 (2004)

${ }^{16}$ T. Gustavsson, B. P. Coto, and E. C. Lim (unpublished)

${ }^{17}$ J.-K. Lee, T. Fujiwara, and E. C. Lim (unpublished).

${ }^{18}$ See Ref. 11 for experimental details.

${ }^{19}$ See, for experimental details, T. Gustavsson, A. Sharonov, and D. Markovitsi, Chem. Phys. Lett. 351, 195 (2002).

${ }^{20}$ S. I. Druzhinin, V. A. Galievsky, and K. A. Zachariasse, J. Phys. Chem. A 109, 11213 (2005).

${ }^{21}$ M. L. Horng, J. A. Gardecki, A. Papazyan, and M. Maroncelli, J. Phys. Chem. 99, 17311 (1995).

${ }^{22}$ Because of the triplet-triplet absorption with intensity maximum at 380 nm [Y. Wang, J. Chem. Soc., Faraday Trans. 2, 1809 (1988)], which strongly overlap the $420 \mathrm{~nm}$ TICT transient, it is very difficult to measure the decay time of the TICT state absorption at $420 \mathrm{~nm}$.

${ }^{23}$ W.-M. Kwok, M. W. George, D. C. Grills, C. Ma, P. Matousek, A. W. Parker, D. Phillips, W. T. Toner, and M. Towrie, Angew. Chem., Int. Ed. 42, 1826 (2003). 